\title{
MicroRNA-29a Promotes Pancreatic Cancer Growth by Inhibiting Tristetraprolin
}

\author{
Xian-Jun Sun ${ }^{a}$ Bing-Yan Liu ${ }^{a}$ Shuo Yan ${ }^{a}$ Ting-Hui Jiang ${ }^{a}$ Hui-Qin Cheng ${ }^{b}$ \\ Hao-Sheng Jiang ${ }^{a}$ Yan $\mathrm{Cao}^{\mathrm{a}} \mathrm{Ai}-\mathrm{Wu} \mathrm{Mao}^{\mathrm{a}}$ \\ aDepartment of Interventional Radiology, Tongren Hospital, Shanghai Jiao Tong University School of

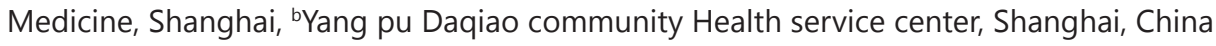

\section{Key Words}

MicroRNA-29a • Tristetraprolin • Pancreatic cancer

\begin{abstract}
Background/Aims: The microRNA (miR) 29 family has been studied extensively for its involvement in several diseases, and aberrant expression of its members is associated with tumorigenesis and cancer progression. Here, we examined the role of miR-29a in pancreatic cancer and the involvement of tristetraprolin (TTP). Methods: We monitored miR-29a and TTP expression in pancreatic cancer by qRT-PCR and western blotting. The effect of miR-29a on pancreatic cancer was determined through MTT assay and migration assay. The results were validated in the tumorigenesis model. Results: We found that miR-29a was up regulated in pancreatic tumor tissues and cell lines and positively correlated with metastasis. Ectopic expression of miR-29a increased the expression of pro-inflammatory factors and epithelialmesenchymal transition (EMT) markers, through down regulating TTP. TTP was down regulated in tumor tissues, and its ectopic expression decreased cell viability and migration in vitro, inhibited tumor growth and the EMT phenotype in vivo, and reversed the effect of miR-29a on tumor cell proliferation and invasion in vitro and in vivo. Conclusion: Our results suggest that miR-29a acts as an oncogene by down regulating TTP and provide the basis for further studies exploring the potential of miR-29a and TTP as biomarkers and targets for the treatment of pancreatic cancer.

\section{Introduction}

Pancreatic cancer is the fourth leading cause of cancer related death, with an estimated 46,420 new cases and 36,000 deaths expected in 2014 in the United States [1]. Although the incidence and mortality of pancreatic cancer have declined slowly in recent years, it remains one of the most aggressive malignancies, with a 5-year relative survival rate of

X.-J. Sun, B.-Y. Liu and S. Yan contributed to the work equally and should be regarded as co-first authors.

Ai-Wu Mao, M.D. and Ting-Hui Jiang, M.D.

\section{KARGER 125}




\section{Cellular Physiology Cell Physiol Biochem 2015;37:707-718

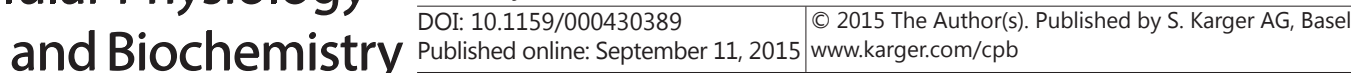 \\ Sun et al.: MiR-29a Promoted Pancreatic Cancer Development}

approximately 5\%. Pancreatic cancer cells have a high potential for invasion and metastasis, and approximately $80 \%$ of patients have metastatic disease at diagnosis, which may account for the high mortality from this disease [2-4]. Improving our understanding of the molecular mechanism(s) involved in the aggressive growth characteristics of pancreatic cancer is important for the design of effective therapeutic strategies.

Tristetraprolin (TTP), also named ZFP36, binds to the AU-rich elements (AREs) at the 3' untranslated region (3'UTR) of target mRNAs, leading to their deadenylation and degradation [5]. The role of TTP in destabilizing target mRNAs was first suggested by its interaction with tumor necrosis factor $\alpha(\mathrm{TNF} \alpha)$ [6]. TTP has been suggested to function as a tumor suppressor based on its down regulation in many human cancers and its negative correlation with tumor progression in breast and prostate cancers [7-8]. The interaction of TTP with ARE-containing mRNAs associated with tumor development and progression, such as c-Myc, and cancer-related inflammation such as TNF $\alpha$, various interleukins, and cyclooxygenase 2 (COX-2), further supports its tumor suppressive role [9-13]. However, the involvement of TTP in pancreatic cancer remains unknown.

MicroRNAs (miRNAs) constitute an evolutionarily conserved class of small (approximately 22 nucleotides in length) noncoding RNA molecules that negatively regulate gene expression by binding to the 3'UTR of target mRNAs resulting in transcript degradation or translational repression [14]. Deregulation of miRNAs is associated with several diseases including cancer, and tumor-associated miRNAs can function as tumor suppressors or oncogenes depending on their target mRNAs $[15,16]$. Many miRNAs including miR-200c, miR-375, miR-10b, miR-181b, miR-196a, and miR-21 have been implicated in pancreatic cancer development [17-19]. The miR-29a family, which consists of three members (miR$29 \mathrm{a}-\mathrm{c}$ ), share a common seed region and therefore target overlapping sets of genes, although its members show different subcellular localization and are differentially regulated [20]. MiR-29a can act both as a tumor suppressor and oncogene, and several targets have been identified and implicated in different malignancies, including chronic lymphocytic leukemia, cholangio-carcinoma and lung cancer [21-23]. TTP was previously identified as a target of miR-29a, and miR-29a mediated down regulation of TTP is associated with epithelialmesenchymal transition (EMT) and metastasis in breast cancer [24].

In the present study, we examined the involvement of miR-29a in pancreatic cancer and its potential role as a regulator of TTP expression. We showed that miR-29a promotes tumor progression and invasion by down regulating TTP in vitro and in vivo, suggesting a novel mechanism of tumorigenesis mediated by the suppression of the oncogenic protein TTP and providing potential biomarkers and therapeutic targets in pancreatic cancer.

\section{Materials and Methods}

\section{Clinical samples and cell lines}

Tumor and adjacent non-tumor tissues were obtained from 30 pancreatic cancer patients treated at Tongren Hospital. And 14 non-metastasis and 16 metastasis samples were included in the current issues.

The human normal pancreatic cell line HPDE6c7 and the pancreatic cancer cell lines Panc-1 and BXPC3 were obtained from ATCC and cultured in Dulbecco's modified Eagle's medium (DMEM) supplemented with 10\% fetal bovine serum (FBS; Life Technologies, Carlsbad, CA, USA), 2 mM glutamine, $100 \mathrm{U} / \mathrm{mL}$ penicillin, and $100 \mu \mathrm{g} / \mathrm{ml}$ streptomycin at $37^{\circ} \mathrm{C}$ in a humidified chamber supplemented with $5 \% \mathrm{CO}_{2}$.

Plasmid construction

Human miR-29a precursor and TTP coding sequences were amplified from human cDNA by PCR with KOD-plus DNA polymerase (TOYOBO, Osaka, Japan). The cycling conditions were 2-step cycle, which was as follows: pre-denaturation: $94^{\circ} \mathrm{C}, 2 \mathrm{~min}$; denaturation: $94^{\circ} \mathrm{C}, 15 \mathrm{sec}$; extension: $68^{\circ} \mathrm{C}, 1 \mathrm{~min} / \mathrm{kb}$; with denaturation and extension steps to be 32 cycles. Primer sequences for PCR amplification were as follows (5'-3'): miR-29a-forward: AAA GGA TCC GCC ATA GAA ACC CAG TTTC, miR-29a-reverse: AAA ACG CGT CCA AGG GAT GAA TGT AATTG; TTP-forward: AAA GGA TCC ATG GCC AAC CGT TAC ACC ATG GATC; TTP-reverse: 


\section{Cellular Physiology Cell Physiol Biochem 2015;37:707-718

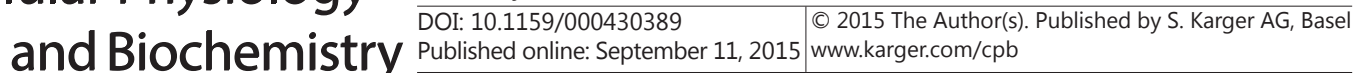 \\ Sun et al.: MiR-29a Promoted Pancreatic Cancer Development}

AAA ACG CGT TCG CTC AGA AAC AGA GAT GCG ATTGA. The amplified sequences were inserted between BamHI-MluI restriction sites in the lentivirus pCDH vector. Virus production in 293T cells was achieved by cotransfecting cells with the lentiviral vector pCDH and packaging plasmid. The supernatant was collected at $48 \mathrm{~h}$ post-transfection and used to generate stably transfected cell lines.

\section{miRNA oligonucleotides and cell transfection}

The synthetic human miR-29a mimics used in these studies were as follows: hsa-miR-29a mimics: $5^{\prime}$-UAGCACCAUCUGAAAUCGGUUA-3'. The antisense oligonucleotides used in these studies were the miR29a inhibitor: 5'- UAA CCG AUU UCA GAU GGU GCUA-3'. These oligonucleotides were transfected into cells using Lipofectamine RNAiMAX kit (Invitrogen, Carlsbad, CA, USA) at 50\% confluence, following the manufacturer's instructions.

\section{Dual-Iuciferase reporter assays}

The TTP $3^{\prime}$ UTR containing the predicted binding site for miR-29a was amplified using primers $5^{\prime}$-CAA AGT GAC TGC CCG GTC AGATC-3' and 5' - CCA GCT TAC ACT CAG ATT GTT TATTT-3'. A fragment of the TTP 3'UTR (1750 bps) and the mutated 3'UTR were inserted into the psiCHECK2 vector. For mutated 3'UTR, the miR-29a putative seed binding site (TGGTGCT) was mutated to the sequence TGACATC by site-directed mutagenesis. The psiCHECK-2 vector containing wild-type (wt) or mutated TTP was transfected into cells with or without the synthetic miR-29a mimic. Thirty-six hours after transfection, luciferase activity was detected using a dual-luciferase reporter assay system and normalized to Renilla activity.

\section{RNA extraction and $q R T-P C R$}

Total RNA was extracted using the TRIzol reagent (life technology) and miRNA expression was analyzed using the TaqMan MicroRNA Assay (Ambion, Austin, TX) to detect mature miRNAs. Experiments were performed using an Applied Biosystems 7900 Real-Time PCR System (ABI, Foster City, CA). To detect the expression of TTP, two-step SYBR Green II qRT-PCR was performed, with GAPDH as endogenous control. Production of complementary DNA with oligo(dT) primers was performed according to the protocol supplied with the PrimerScript ${ }^{\mathrm{RT}}$ Reagent (TaKaRa, Tokyo, Japan). The PCR primers for qRT-PCR assay were as follows $\left(5^{\prime}-3^{\prime}\right)$ :

TTP-forward, GACTGAGCTATGTCGGACCTT,

TTP-reverse, GAGTTCCGTCTTGTATTTGGGG;

TNF $\alpha$-forward, CCTCTCTCTAATCAGCCCTCTG,

TNF $\alpha$-reverse, GAGGACCTGGGAGTAGATGAG;

COX-2-forward, CCTTCCTCCTGTGCCTGATG,

COX-2-reverse, ACAATCTCATTTGAATCAGGAAGCT;

IL-6-forward, ACTCACCTCTTCAGAACGAATTG,

IL-6-reverse, CCATCTTTGGAAGGTTCAGGTTG;

IL-8-forward, TTTTGCCAAGGAGTGCTAAAGA,

IL-8-reverse, AACCCTCTGCACCCAGTTTTC;

GAPDH-forward, GGAGCGAGATCCCTCCAAAAT,

GAPDH-reverse, GGCTGTTGTCATACTTCTCATGG.

All experiments were performed three times in triplicate.

\section{Western blotting}

Pancreatic cancer cells were lysed with lysis buffer (1\% SDS, $50 \mathrm{mM}$ Tris-HCl (pH 6.8), $10 \mathrm{mM}$ dithiothreitol, $10 \%$ glycerol, $0.002 \%$ bromophenol blue, supplemented with a protease inhibitor mixture (Roche, Basel, Switzerland). After quantification, equal amounts of protein were separated by SDS-PAGE, transferred to polyvinylidene difluoride membranes (Millipore, Billerica, MA, USA), immunoblotted with primary antibodies, and visualized with horseradish-peroxidase-coupled secondary antibodies. Primary antibodies were as follows: anti-TTP (Santa Cruz Biotechnology, Santa Cruz, CA, USA); anti-E-cadherin, -N-cadherin, and -vimentin (BD Biosciences, San Diego, CA, USA); and anti-GAPDH (Cell Signaling Technology, Danvers, MA, USA). 


\section{Cellular Physiology Cell Physiol Biochem 2015;37:707-718

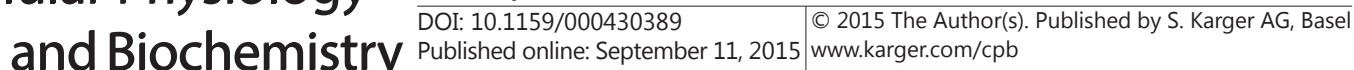 \\ Sun et al.: MiR-29a Promoted Pancreatic Cancer Development}

MTT assay

Cell proliferation was examined with the 3-(4, 5-dimethylthiazol-2-yl)-2, 5-diphenyltetrazolium bromide (MTT) assay. After $6 \mathrm{~h}$ of transfection, cells were seeded into 96-well microtiter plates. After seeding for 1, 2, 3 and $4 \mathrm{~d}, 20 \mu \mathrm{L}$ of MTT solution was added into each well. The optical density (OD) values were measured at $490 \mathrm{~nm}$ using the scan reader (Labsystems, Santa Fe, NM, USA). Each assay was performed three times and the average was calculated.

Migration assay

Transwell chambers (24-well insert; Corning, NY, USA) were used to for cell migration assays. After transfection, cells were suspended in medium without serum and added to the upper chambers. After migration for $12 \mathrm{~h}$, cells on the upper membrane surface were removed. The lower membrane surface was fixed with $4 \%$ formaldehyde, stained with Hoechst and counted under a fluorescence microscope. And 5-6 random fields were collected for quantification.

Tumorigenesis in nude mice

The supernatants of pCDH lentiviruses harboring miR-29a or TTP were produced and collected as described above. Pancreatic cancer cells were subjected to infection of indicated lentiviruses respectively for $24 \mathrm{~h}$, with the lentivirus from pCDH empty vector as the control. As pCDH vector harbored puromycin resistance gene, stable expression cell line was obtained by puromycin screening. Pancreatic cancer cells $\left(1 \times 10^{6}\right)$ stably expressing miR-29a or TTP by lentivirus were collected and inoculated subcutaneously into the right flank region of 4-week-old male BALB/c nude mice (Institute of Zoology, Chinese Academy of Sciences, Shanghai, China). Tumor nodules were measured weekly with a caliper. Mice were sacrificed at the end of one month and tumor growth rate and rate of inhibition were calculated. Three independent experiments were performed for each experimental group.

\section{Statistics}

Statistical analysis was performed using SPSS15.0. Values were expressed as mean \pm standard deviation (s.d.) for parametric data. Differences between groups were calculated using the Student's t-test and non-parametric test (Mann-Whitney U-test). A $p$ value of $<0.05$ was defined as statistically significant.

\section{Results}

miR-29a is up regulated in pancreatic cancer tissues and cell lines and associated with metastasis

The expression of miR-29a was measured in 30 paired tumor and adjacent non-tumor tissues from patients with pancreatic cancer using qRT-PCR. MiR-29a was significantly up regulated in pancreatic cancer tissues compared with adjacent tumor tissues (Fig. 1A) and significantly higher in patients with metastatic than in those with non-metastatic disease ( $p<0.05$, both; Fig. 1B). Assessment of miR-29a expression in cultured cells by qRT-PCR showed that miR-29a was significantly up regulated in the pancreatic cancer cell lines Panc-1 and BxPC-3 compared to the normal pancreatic ductal epithelial cell line HPDE6c7 $(p<0.05$, Fig. 1C).

Effect of miR-29a levels on the proliferation and migratory ability of pancreatic cancer cells

To examine the effect of miR-29a expression on cell viability, pancreatic cancer cells were transfected with miR-control, miR-29a mimics or anti-miR-29a, and cells were analyzed using the MTT and Transwell migration assays. Ectopic expression of miR-29a in the Panc-1 and BxPC-3 cell lines significantly increased cell viability in a time-dependent manner (Fig. 2A) and increased migratory ability (Fig. 2B) compared to miR-control transfected cells ( $p<$ 0.05 all). Conversely, inhibition of miR-29a significantly decreased the viability and migratory ability of Panc- 1 and BxPC-3 cells compared to miR-control transfected cells (Fig. 2C and D). 
A

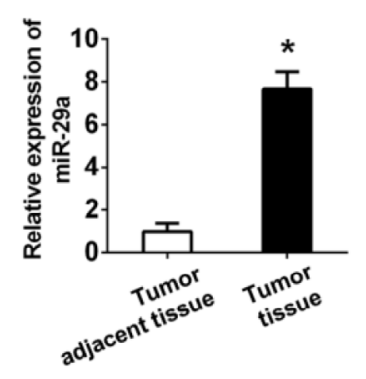

B

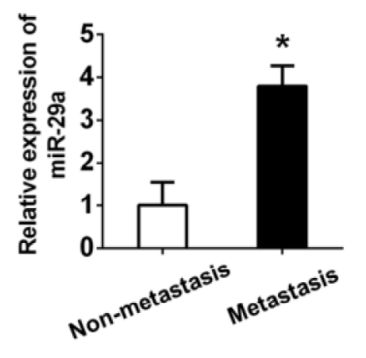

C

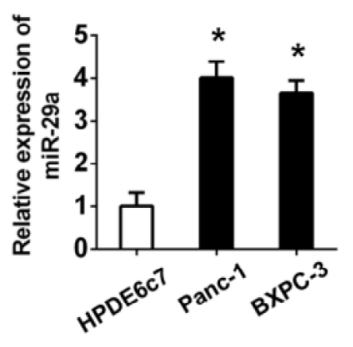

Fig. 1. Expression of miR-29a in pancreatic cancer cells. (A and B) miR-29a expression was measured in human pancreatic cancer tissues and normalized to that in adjacent non-tumor tissues, (A) and in metastasis and non-metastasis associated tumor samples (B) by qRT-PCR. (C) miR-29a expression was measured in the normal pancreatic cell line HPDE6c7 and in the pancreatic cancer cell lines Panc-1 and BXPC-3 by qRT-PCR. Data were normalized to the miR-29a expression of HPDE6c7 cells. *, $p<0.05$.

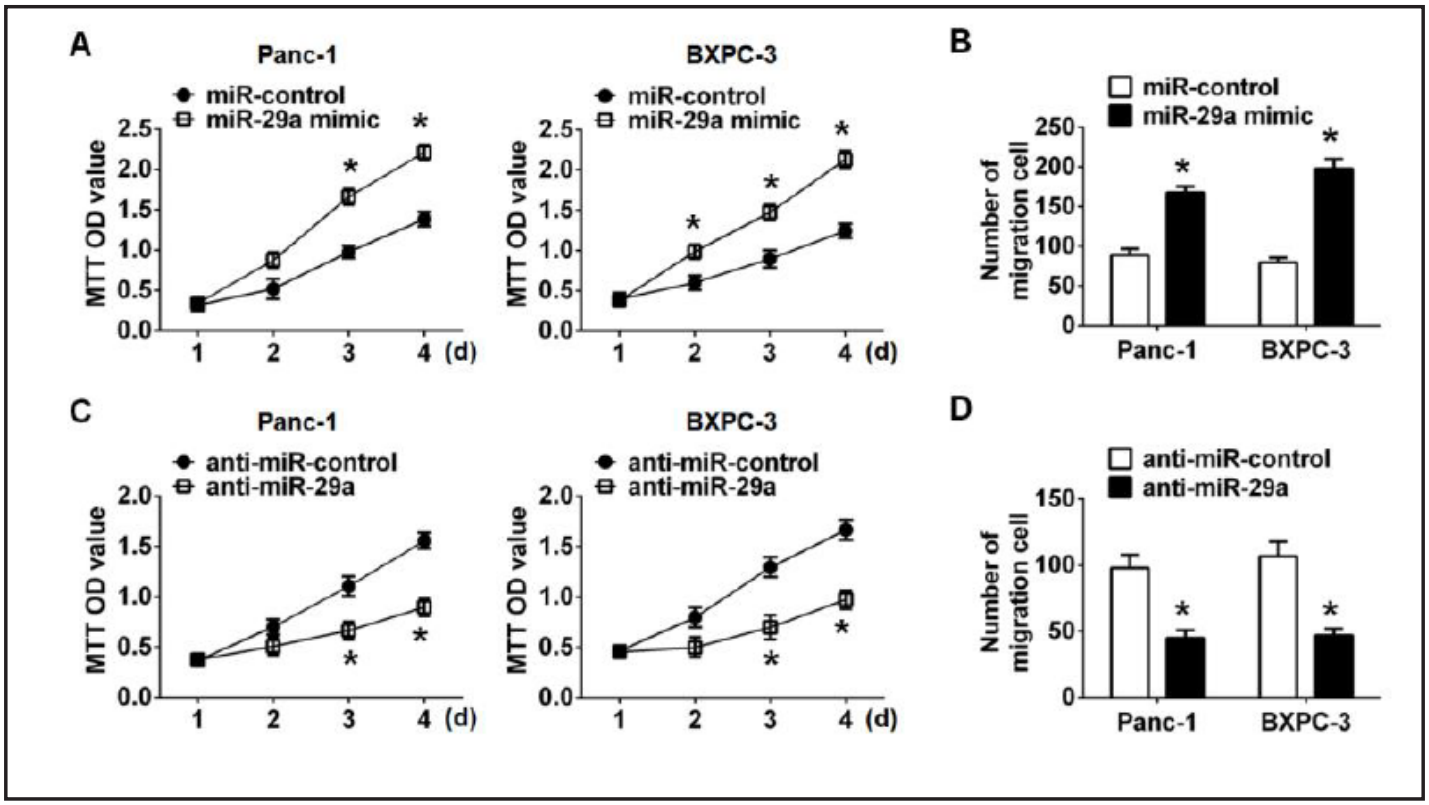

Fig. 2. miR-29a promotes tumor proliferation and migration. (A and B) Cell viability was measured using the MTT assay in Panc-1 and BXPC-3 cells transfected with miR-29a mimics or miR-control on days 1, 2, 3, and 4 after seeding (A) and cell migration was assessed using the Transwell assay (B). (C and D) Panc-1 and BXPC3 cells were transfected with antisense oligonucleotides against miR-29a (anti-miR-29a) or anti miR-control and cell viability was assessed using the MTT assay on days 1, 2, 3, and 4 after seeding (C), and cell migration was assessed using the Transwell assay (D). *,$p<0.05$.

MiR-29a induces EMT and the expression of pro-inflammatory factors by targeting TTP

Because miR-29a overexpression plays a role in EMT and metastasis through the down regulation of TTP in breast cancer [24], we examined the effect of miR-29a overexpression on EMT and the expression of pro-inflammatory factors in pancreatic cancer cells. To confirm TTP as a direct target of miR-29a, the wild-type or a mutant 3'UTR of TTP was transfected into Panc- 1 and BxPC-3 cells in the presence or absence of miR-29a mimics. Measurement of luciferase activity showed that ectopic expression of miR-29a significantly decreased the transcriptional activity of the wild-type but not that of the mutant TTP 3 'UTR in both cell lines (Fig. 3A), confirming that TTP is a direct target of miR-29a. Western blot analysis showed that ectopic expression of miR-29a down regulated TTP and the expression of the epithelial 


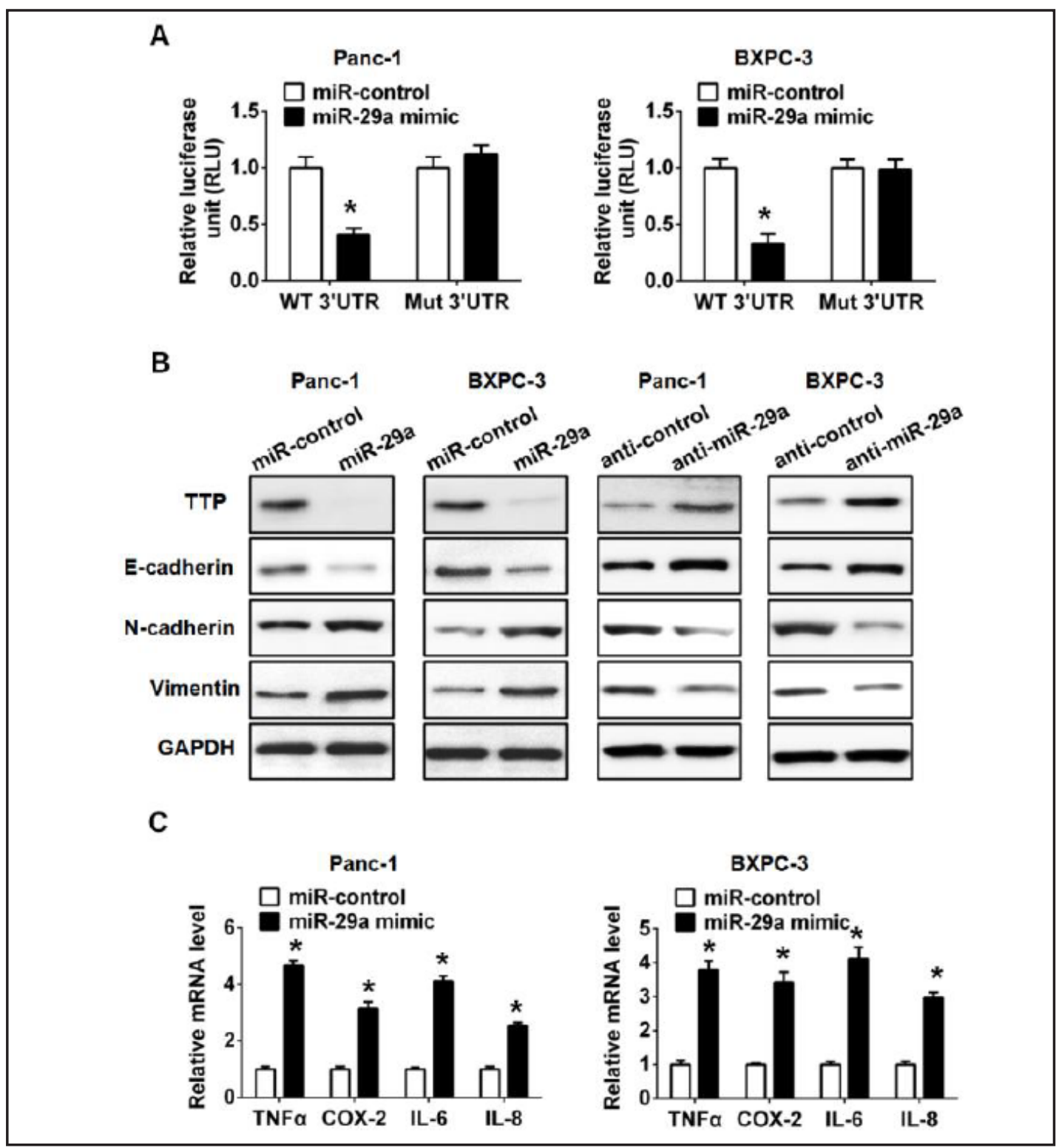

Fig. 3. miR-29a modulates the expression of TTP by direct binding. (A) Panc-1 and BXPC-3 cells were co-transfected with a luciferase reporter plasmid containing the wild-type (wt) or mutant (mut) 3'UTR of TTP in the presence or absence of miR-29a mimics. Luciferase activity was determined $36 \mathrm{~h}$ after transfection. Data were normalized to the luciferase activity of cells transfected with miR-control. (B) The expression of TTP and markers of EMT was assessed by western blotting in the indicated cells. (C) The expression of TNF $\alpha$, COX-2, IL-6, IL-8 was assessed by qRT-PCR in cells transfected with miR-29a mimics and normalized to that in miR-control transfected cells. *, $p<0.05$, $\mathrm{n}=3$.

marker E-cadherin and up regulated the mesenchymal markers $\mathrm{N}$-cadherin and vimentin, whereas miR-29a inhibition had the opposite effects (Fig. 3B), indicating that miR-29a plays a role in EMT in pancreatic cancer. Ectopic expression of miR-29a significantly promoted the expression of the pro-inflammatory factors TNF $\alpha, \mathrm{COX}-2$, interleukin (IL)- 6 and IL-8 in Panc1 and BxPC-3 cells (Fig. 3C). Taken together with the known anti-inflammatory role of TTP [5] and our luciferase assay results, these findings indicate that the pro-inflammatory effect of miR-29a is mediated by the down regulation of TTP in pancreatic cancer cells.

\section{KARGER}




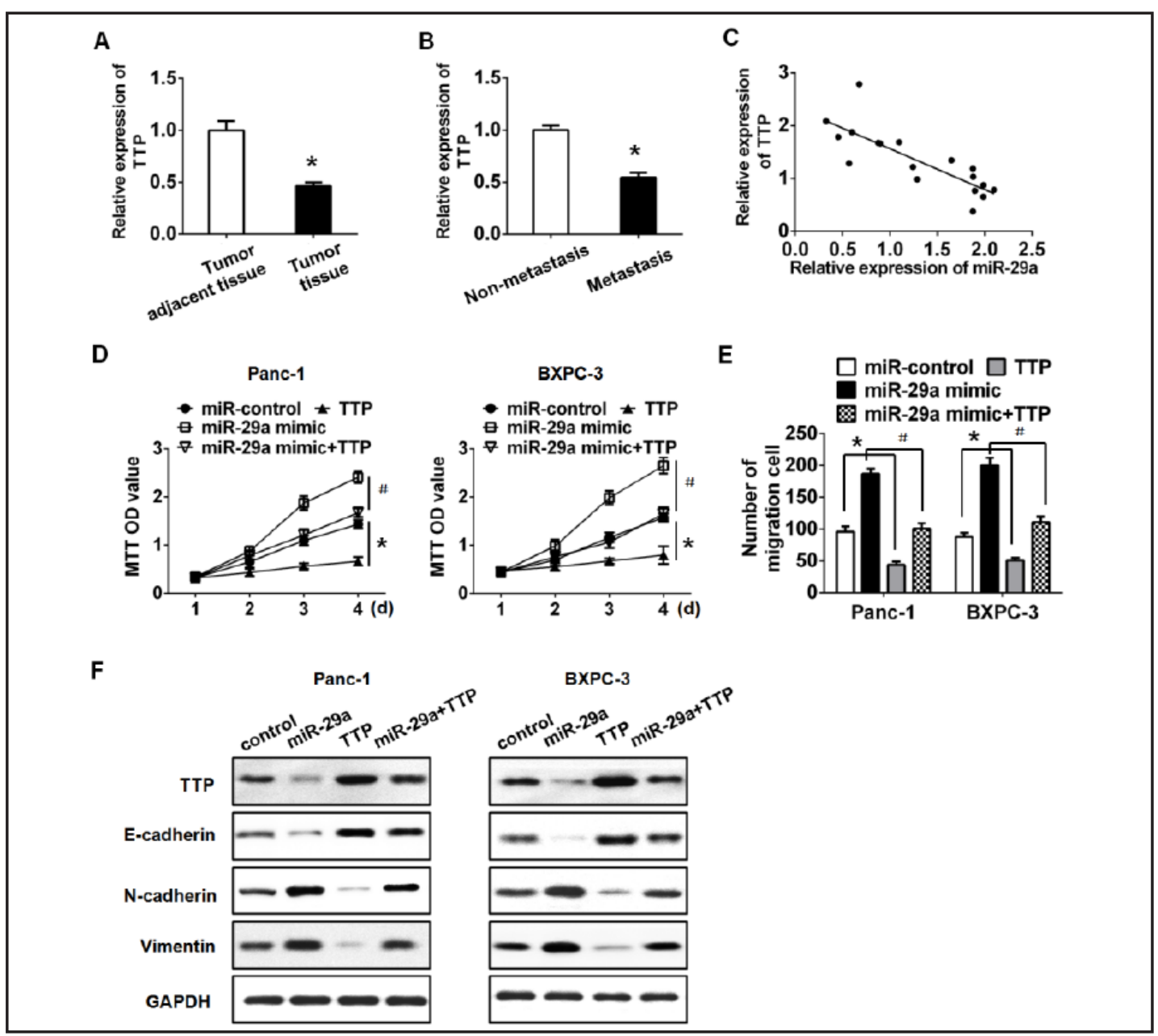

Fig. 4. TTP overexpression rescues the effect of miR-29a on pancreatic cancer development. (A and B) The expression of TTP was assessed in human pancreatic cancer and adjacent non-tumor tissues (A) and in metastasis and non-metastasis associated tissue samples (B) by qRT-PCR and expressed as relative expression. (C) Correlation between miR-29a and TTP protein expression in tumor samples. (D and E) Panc-1 and BXPC-3 cells overexpressing miR-29a, TTP, or both was determined using the MTT assay (D) and migration was assessed using the Transwell assay (E). (F) The expression of EMT markers was assessed by western blotting in miR-29a or TTP expressing cells. * and \#, $p<0.05$.

The effect of miR-29a on pancreatic cancer cell viability and invasion is mediated by the down regulation of TTP

To further examine the involvement of TTP in the effect of miR-29a in pancreatic cancer, TTP expression was measured in patient samples by qRT-PCR, which showed that TTP was significantly down regulated in tumor tissues compared to adjacent non-tumor tissues (Fig. 4A) and in metastatic compared to non-metastatic disease (Fig. 4B) ( $p<0.05$ both). Figure 4C is a plot of the relative expression of TTP and miR-29a, which shows an inverse correlation between TTP and miR-29a expression in pancreatic tumors. Importantly, ectopic expression of TTP decreased the viability and migratory ability of Panc- 1 and BXPC- 3 cells in a time dependent manner and reversed the miR-29a induced increase of cell viability and migration, restoring values to control levels (Fig. 4D and E). Overexpression of TTP up regulated the epithelial marker E-cadherin and down regulated the mesenchymal markers $\mathrm{N}$-cadherin and vimentin, and reversed the miR-29a mediated induction of EMT in Panc-1 and BXPC-3 cells (Fig. 4F). Taken together, these results indicated that the effect of miR-29a 
Fig. 5. Xenograft mouse tumor model. Xenograft tumors were generated by subcutaneous injection of control, miR-29a overexpressing, TTP overexpressing or miR-29a and TTP expressing Panc- 1 and BXPC3 cells. (A) Representative images of tumors isolated from nude mice on day 28. (B) Tumor volumes were measured at the indicated times. (C) The expression of EMT markers in xenograft tumors was determined by western blotting. For miR29a mimic group vs. control group: ${ }^{*}, p<0.05$; for miR-29a mimic+TTP group vs. miR-29a mimic group: \#, $p<0.05$.
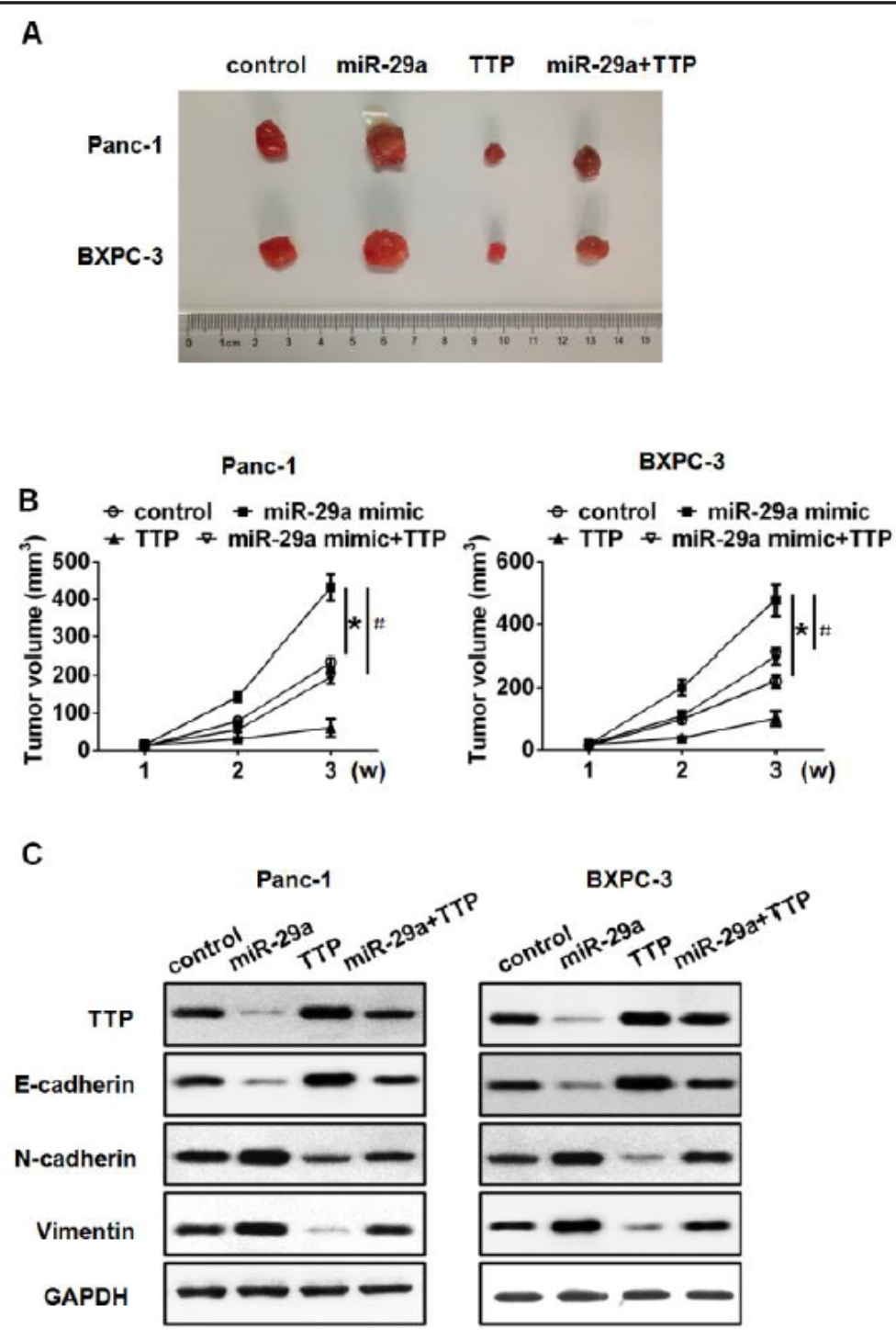

on cell viability, invasion and EMT is mediated by the down regulation of its target TTP in pancreatic cancer cells.

MiR-29a promotes pancreatic xenograft tumor growth and invasion by down regulating TTP

To examine the effect of miR-29a in vivo, a xenograft model was generated by inoculating nude mice with pancreatic cancer cells stably expressing miR-29a or TTP by lentivirus. The stable cells were produced by lentivirus infection, with puromycin screening to generate stable cell lines. Tumor volume was measured weekly and the results are plotted in Fig. 5B, which shows that miR-29a overexpressing tumors grew significantly faster than control tumors, whereas TTP overexpression significantly inhibited tumor growth. Tumors coexpressing miR-29a and TTP showed similar growth than control tumors, indicating that TTP overexpression rescued the effect of miR-29a. Fig. 5A shows representative images of tumors collected on day 28, which shows that miR-29a overexpression promoted tumor growth and TTP restored normal growth, reversing the effect of miR-29a. Western blot assessment of EMT markers in lysates prepared from xenograft tumors showed that TTP and E-cadherin were down regulated and $\mathrm{N}$-cadherin and vimentin were up regulated in miR-29a overexpressing tumors (Fig. 5C). TTP overexpressing tumors showed the inverse 


\section{Cellular Physiology Cell Physiol Biochem 2015;37:707-718

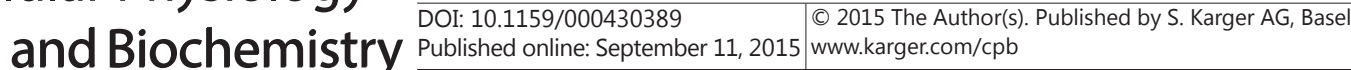 \\ Sun et al.: MiR-29a Promoted Pancreatic Cancer Development}

correlation between epithelial and mesenchymal markers, indicating the suppression of EMT. TTP reversed the effect of miR-29a on promoting EMT, indicating that miR-29a induced EMT in pancreatic cancer xenograft tumors by down regulating TTP.

\section{Discussion}

Although the prevalence of pancreatic cancer is relatively low, the mortality from this disease is extremely high, and the majority of patients are diagnosed at advanced/metastatic stages of the disease, underscoring the need to identify diagnostic and prognostic markers to improve the management of patients with this disease. MiRNAs regulate many cellular processes, and because of their association with tumor development and progression, they have been studied extensively as biomarkers in cancer [25]. In pancreatic cancer, several up regulated or down regulated miRNAs have been identified, suggesting that miRNA expression profiles can discriminate normal from cancerous pancreatic tissues [19]. In the present study, we examined the role of miR-29a in pancreatic cancer. We showed that miR29a is up regulated in pancreatic cancer tissues and cell lines and associated with metastasis, and its overexpression promoted tumor cell proliferation and invasion in vitro and in vivo, suggesting that it plays an oncogenic role. The miR-29 family was widely expressed and previously linked to pancreatic cancer $[26,27]$. In a miRNA array comparing pancreatic cancer samples to a normal pancreatic tissue set, miR-29c was down regulated by more than five-fold in pancreatic cancer samples [27]. MiR-29a was shown to play a role in the response of pancreatic cancer cells to treatment by inducing resistance to gemcitabine via activation of the Wnt/ $\beta$-catenin pathway in pancreatic cancer cell lines [28]. MiR-29a activates Wnt signaling, which plays an important role in pancreatic cancer, by downregulating the negative regulators Dikkopf-1, Kremen 2 and secreted frizzled related protein 2 in human osteoblasts [29]. MiR-29a has been shown to act both as an oncogene and a tumor suppressor miRNA. MiR-29a promotes apoptosis and represses hepatocellular carcinoma tumorigenicity [30]. In cervical squamous cell carcinoma, miR-29a acts as a tumor suppressor and inhibits migration and invasion by targeting heat shock protein 47 [31]. However, in acute myeloid leukemia, miR-29a is overexpressed and plays an oncogenic role by accelerating G1 to S/ G2 cell cycle progression, whereas down regulation of miR-29 plays a role in mantle cell lymphoma through its effect on CDK6 expression $[32,33]$. These studies demonstrate that the function of miR-29 in cancer may be context dependent and reveal the role of the miR-29 family in pancreatic cancer, as indicated by the results of the present study.

Based on a previous study that identified TTP mRNA as a target of miR-29a in breast cancer and extensive research linking TTP suppression to the exacerbation of tumorigenic phenotypes, we examined the association between miR-29a and TTP in pancreatic cancer. Our findings verified TTP as a target of miR-29a and showed that miR-29a down regulates TTP in association with the induction of EMT and the up regulation of pro-inflammatory factors, whereas ectopic expression of TTP rescued the effect of miR-29a in vitro and in a xenograft mouse model in vivo, confirming that miR-29a acts as an oncomir via its target TTP in pancreatic cancer. Although the effects of TTP down regulation on cancer development and progression have been documented extensively, the mechanisms regulating TTP expression and how its loss enhances tumorigenesis remain unclear. TTP activity is regulated by phosphorylation through the p38 MAPK and ERK MAPK pathways, and in melanoma cells, ERK suppresses TTP levels by targeting the protein for proteasomal degradation [34, 35]; however, few mechanisms of TTP down regulation have been reported to date. Sohn et al. showed an epigenetic mechanism of TTP regulation via methylation of a CpG site in the TTP promoter [36], and TTP was shown to be down regulated by miR-29a in breast cancer [24, 27]. Given the known role of TTP in inflammation and cancer, our in vitro and in vivo findings showing the modulation of its expression by miR-29a in association with pancreatic cancer cell proliferation and invasion are of particular importance. Future experiments should be aimed at exploring the effect of miR-29a on other cancer-related TTP targets in pancreatic 


\section{Cellular Physiology Cell Physiol Biochem 2015;37:707-718

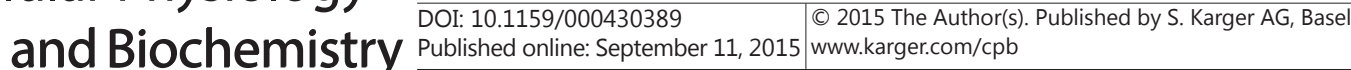 \\ Sun et al.: MiR-29a Promoted Pancreatic Cancer Development}

cancer, such as mRNAs encoding angiogenic inducers including VEGF, and invasion related proteins such as matrix metalloproteinase 1 and the urokinase plasminogen activator (uPA) and its co-activating receptor uPAR, and the hypoxia-related factors [37].

Although many drugs have been applied in pancreatic cancer treatment [38, 39], the mortality of pancreatic cancer is still high. Therefore, it is urgent to develop new targets for cancer intervention. In the present study, we showed that miR-29a is up regulated and TTP is down regulated in pancreatic cancer cell lines and tissues, and miR-29a promotes tumor cell proliferation and the EMT phenotype by modulating TTP expression. The present findings linking miR-29a to the regulation of TTP expression in pancreatic cancer identify potential novel biomarkers and therapeutic targets and provide the basis for future studies aimed at exploring the effects of the modulation of TTP expression as a potential therapeutic strategy in cancer.

\section{Acknowledgements}

Our study was supported by the Research Grant of Xinglin Nova Project of Shanghai Municipal Commission of Health and Family Planning (NO.ZY3-RCPY-2-2083); the Key Medical Disciplines of Shanghai Municipal (NO. ZK2012A04); Research Grant of Shanghai Municipal Commission of Health and Family Planning (NO. 20124178); Research Grant for Traditional Chinese Medicine of Shanghai Municipal Commission of Health and Family Planning (NO.2014LP022A); the Key Project of Shanghai Municipal Commission of Health and Family Planning Supporting Programs (NO.20134036); and The Research Grant of Changning District Commission of Health and Family Planning of Shanghai (N0.20144Y015).

\section{Disclosure Statement}

All authors have read the manuscript and agreed with the publication. No potential conflicts of interest relevant to this article were reported.

\section{References}

1 Siegel R, Ma J, Zou Z, Jemal A: Cancer statistics, 2014. CA Cancer J Clin 2014;64:9-29.

2 Huang C, Xie K: Analysis of the potential for pancreatic cancer metastasis in vitro and in vivo. Methods Mol Biol 2013;980:301-319.

3 Siegel R, Naishadham D, Jemal A: Cancer statistics, 2013. CA Cancer J Clin 2013;63:11-30.

4 Jones OP, Melling JD, Ghaneh P: Adjuvant therapy in pancreatic cancer. World J Gastroenterol 2014;20:14733-14746.

5 Sanduja S, Blanco FF, Young LE, Kaza V, Dixon DA: The role of tristetraprolin in cancer and inflammation. Front Biosci (Landmark Ed) 2012;17:174-188.

6 Taylor GA, Carballo E, Lee DM, Lai WS, Thompson MJ, Patel DD, Schenkman DI, Gilkeson GS, Broxmeyer HE, Haynes BF, Blackshear PJ: A pathogenetic role for tnf alpha in the syndrome of cachexia, arthritis, and autoimmunity resulting from tristetraprolin (ttp) deficiency. Immunity 1996;4:445-454.

7 Brennan SE, Kuwano Y, Alkharouf N, Blackshear PJ, Gorospe M, Wilson GM: The mrna-destabilizing protein tristetraprolin is suppressed in many cancers, altering tumorigenic phenotypes and patient prognosis. Cancer Res 2009;69:5168-5176.

8 Carrick DM, Blackshear PJ: Comparative expression of tristetraprolin (ttp) family member transcripts in normal human tissues and cancer cell lines. Arch Biochem Biophys 2007;462:278-285.

9 Marderosian M, Sharma A, Funk AP, Vartanian R, Masri J, Jo OD, Gera JF: Tristetraprolin regulates cyclin d1 and c-myc mrna stability in response to rapamycin in an akt-dependent manner via p38 mapk signaling. Oncogene 2006;25:6277-6290. 


\section{Cellular Physiology Cell Physiol Biochem 2015;37:707-718 and Biochemistry POI: 10.1159/000430389 2015 (c) 2015 The Author(s). Published by S. Karger AG, Basel \\ Sun et al.: MiR-29a Promoted Pancreatic Cancer Development}

10 Van Tubergen E, Vander Broek R, Lee J, Wolf G, Carey T, Bradford C, Prince M, Kirkwood KL, D'Silva NJ: Tristetraprolin regulates interleukin-6, which is correlated with tumor progression in patients with head and neck squamous cell carcinoma. Cancer 2011;117:2677-2689.

11 Zhao W, Liu M, D'Silva NJ, Kirkwood KL: Tristetraprolin regulates interleukin-6 expression through p38 mapk-dependent affinity changes with mrna 3' untranslated region. J Interferon Cytokine Res 2011;31:629-637.

12 Boutaud O, Dixon DA, Oates JA, Sawaoka H: Tristetraprolin binds to the cox-2 mrna 3' untranslated region in cancer cells. Adv Exp Med Biol 2003;525:157-160.

13 Ross CR, Brennan-Laun SE, Wilson GM: Tristetraprolin: Roles in cancer and senescence. Ageing Res Rev 2012;11:473-484.

14 Ma C, Nong K, Wu B, Dong B, Bai Y, Zhu H, Wang W, Huang X, Yuan Z, Ai K: Mir-212 promotes pancreatic cancer cell growth and invasion by targeting the hedgehog signaling pathway receptor patched-1. J Exp Clin Cancer Res 2014;33:54.

15 Wang D, Qiu C, Zhang H, Wang J, Cui Q Yin Y: Human microrna oncogenes and tumor suppressors show significantly different biological patterns: From functions to targets. PLoS One 2010;5: e13067

16 Esquela-Kerscher A, Slack FJ: Oncomirs - micrornas with a role in cancer. Nat Rev Cancer 2006;6:259-269.

17 Pai P, Rachagani S, Are C, Batra SK: Prospects of mirna-based therapy for pancreatic cancer. Curr Drug Targets 2013;14:1101-1109.

18 Frampton AE, Fletcher CE, Gall TM, Castellano L, Bevan CL, Stebbing J, Krell J: Circulating peripheral blood mononuclear cells exhibit altered mirna expression patterns in pancreatic cancer. Expert Rev Mol Diagn 2013;13:425-430.

19 Humeau M, Torrisani J, Cordelier P: Mirna in clinical practice: Pancreatic cancer. Clin Biochem 2013;46:933-936.

20 Kriegel AJ, Liu Y, Fang Y, Ding X, Liang M: The mir-29 family: Genomics, cell biology, and relevance to renal and cardiovascular injury. Physiol Genomics 2012;44:237-244.

21 Pekarsky Y, Santanam U, Cimmino A, Palamarchuk A, Efanov A, Maximov V, Volinia S, Alder H, Liu CG, Rassenti L, Calin GA, Hagan JP, Kipps T, Croce CM: Tcl1 expression in chronic lymphocytic leukemia is regulated by mir-29 and mir-181. Cancer Res 2006;66:11590-11593.

22 Fabbri M, Garzon R, Cimmino A, Liu Z, Zanesi N, Callegari E, Liu S, Alder H, Costinean S, FernandezCymering C, Volinia S, Guler G, Morrison CD, Chan KK, Marcucci G, Calin GA, Huebner K, Croce CM: Microrna-29 family reverts aberrant methylation in lung cancer by targeting DNA methyltransferases 3a and 3b. Proc Natl Acad Sci U S A 2007;104:15805-15810.

23 Mott JL, Kobayashi S, Bronk SF, Gores GJ: Mir-29 regulates mcl-1 protein expression and apoptosis. Oncogene 2007;26:6133-6140.

24 Gebeshuber CA, Zatloukal K, Martinez J: Mir-29a suppresses tristetraprolin, which is a regulator of epithelial polarity and metastasis. EMBO Rep 2009;10:400-405.

25 Iorio MV, Croce CM: Microrna dysregulation in cancer: Diagnostics, monitoring and therapeutics. A comprehensive review. EMBO Mol Med 2012;4:143-159.

26 Melo SF, Fernandes T, Barauna VG, Matos KC, Santos AA, Tucci PJ, Oliveira EM: Expression of microrna-29 and collagen in cardiac muscle after swimming training in myocardial-infarcted rats. Cell Physiol Biochem 2014;33:657-669.

27 Szafranska AE, Davison TS, John J, Cannon T, Sipos B, Maghnouj A, Labourier E, Hahn SA: Microrna expression alterations are linked to tumorigenesis and non-neoplastic processes in pancreatic ductal adenocarcinoma. Oncogene 2007;26:4442-4452.

28 Nagano H, Tomimaru Y, Eguchi H, Hama N, Wada H, Kawamoto K, Kobayashi S, Mori M, Doki Y: Microrna29a induces resistance to gemcitabine through the wnt/beta-catenin signaling pathway in pancreatic cancer cells. Int J Oncol 2013;43:1066-1072.

29 Kapinas K, Kessler C, Ricks T, Gronowicz G, Delany AM: Mir-29 modulates wnt signaling in human osteoblasts through a positive feedback loop. J Biol Chem 2010;285:25221-25231.

30 Xiong Y, Fang JH, Yun JP, Yang J, Zhang Y, Jia WH, Zhuang SM: Effects of microrna-29 on apoptosis, tumorigenicity, and prognosis of hepatocellular carcinoma. Hepatology 2010;51:836-845.

31 Yamamoto N, Kinoshita T, Nohata N, Yoshino H, Itesako T, Fujimura L, Mitsuhashi A, Usui H, Enokida H, Nakagawa M, Shozu M, Seki N: Tumor-suppressive microrna-29a inhibits cancer cell migration and invasion via targeting hsp47 in cervical squamous cell carcinoma. Int J Oncol 2013;43:1855-1863. 


\section{Cellular Physiology Cell Physiol Biochem 2015;37:707-718 \begin{tabular}{ll|l|l|l|l} 
DOI: 10.1159/000430389 2015 The Author(s). Published by S. Karger AG, Basel \\
and Biochemistry
\end{tabular} \\ Sun et al.: MiR-29a Promoted Pancreatic Cancer Development}

32 Han YC, Park CY, Bhagat G, Zhang J, Wang Y, Fan JB, Liu M, Zou Y, Weissman IL, Gu H: Microrna-29a induces aberrant self-renewal capacity in hematopoietic progenitors, biased myeloid development, and acute myeloid leukemia. J Exp Med 2010;207:475-489.

33 Zhao JJ, Lin J, Lwin T, Yang H, Guo J, Kong W, Dessureault S, Moscinski LC, Rezania D, Dalton WS, Sotomayor E, Tao J, Cheng JQ: Microrna expression profile and identification of mir-29 as a prognostic marker and pathogenetic factor by targeting cdk6 in mantle cell lymphoma. Blood 2010;115:2630-2639.

34 Marchese FP, Aubareda A, Tudor C, Saklatvala J, Clark AR, Dean JL: Mapkap kinase 2 blocks tristetraprolindirected mrna decay by inhibiting caf1 deadenylase recruitment. J Biol Chem 2010;285:27590-27600.

35 Bourcier C, Griseri P, Grepin R, Bertolotto C, Mazure N, Pages G: Constitutive erk activity induces downregulation of tristetraprolin, a major protein controlling interleukin8/cxcl8 mrna stability in melanoma cells. Am J Physiol Cell Physiol 2011;301:C609-618.

36 Sohn BH, Park IY, Lee JJ, Yang SJ, Jang YJ, Park KC, Kim DJ, Lee DC, Sohn HA, Kim TW, Yoo HS, Choi JY, Bae YS, Yeom YI: Functional switching of tgf-beta1 signaling in liver cancer via epigenetic modulation of a single cpg site in ttp promoter. Gastroenterology 2010;138:1898-1908.

37 Shi CY, Fan Y, Liu B, Lou WH: Hif1 contributes to hypoxia-induced pancreatic cancer cells invasion via promoting qsox1 expression. Cell Physiol Biochem 2013;32:561-568.

38 Melo SF, Fernandes T, Barauna VG, Matos KC, Santos AA, Tucci PJ, Oliveira EM: Expression of microrna-29 and collagen in cardiac muscle after swimming training in myocardial-infarcted rats. Cell Physiol Biochem 2014;33:657-669.

39 Shi CY, Fan Y, Liu B, Lou WH: Hif1 contributes to hypoxia-induced pancreatic cancer cells invasion via promoting qsox1 expression. Cell Physiol Biochem 2013;32:561-568. 